\title{
Biogenic Organic/Inorganic Nano-Composite: Imaging the Macro to Atomic Structure of Herdmania momus Vateritic Spicules
}

Lee Kabalah-Amitai ${ }^{1,2}$, Boaz Mayzel ${ }^{3}$, Yaron Kauffmann ${ }^{1}$, Andrew N. Fitch ${ }^{4}$, Leonid Bloch $^{1}$, Pupa U. P. A. Gilbert ${ }^{5}$, and Boaz Pokroy ${ }^{1,2}$.

1. Department of Materials Science \& Engineering, Technion - Israel Institute of Technology, 32000 Haifa, Israel

2. Russell Berrie Nanotechnology Institute, Technion - Israel Institute of Technology, 32000 Haifa, Israel

${ }^{3 .}$ Department of Biology, Tel Aviv University, 69978 Tel Aviv, Israel

4. European Synchrotron Radiation Facility, BP 220, F-38043 Grenoble Cedex, France

5. Department of Physics, University of Wisconsin, Madison, 1150 University Avenue, Madison, Wisconsin 53706, USA

Biogenic minerals are known, and most of them have been extensively studied because of their fascinating properties[1]. By far the most abundant biogenic minerals are calcium carbonates, which exist in different polymorphs: calcite, aragonite, vaterite, and amorphous $\mathrm{CaCO}_{3}$, listed in order of decreasing thermodynamic stability under normal conditions, or of increasing solubility. Biogenic vaterite is extremely rare and in biominerals, vaterite occurs as a minority-component of a larger formed structure or as the result of a defective biological process. The only known example of a completely vateritic mineralized structure is the spicule of the solitary ascidian, Herdmania momus. In addition, despite considerable research on vaterite synthesis, and although vaterite structure has been studied since the beginning of the last century, the crystallographic structure of this $\mathrm{CaCO}_{3}$ crystal is still a matter of debate.

Herdmania momus specimens were collected by SCUBA diving from marine floats in Eilat at the northern tip of the Gulf of Aqaba (Red Sea), Israel. Specimens were cut into small tissue pieces, which were then treated with $7 \%$ sodium hypochlorite $(\mathrm{NaOCl})$ to remove the organic matter. $\mathrm{NaOCl}$ was replaced frequently and vials were shaken gently for 6 weeks until all organic matter was removed. Spicules were filtered to remove debris and washed 3 times in $100 \%$ ethanol.

In this work, state-of-the-art techniques such as synchrotron X-ray diffraction, synchrotron micro- and nanotomography and aberration-corrected Transmission Electron Microscopy were used for characterizing the structure of these spicules in all scales from the micron-size to the atomic level. Sample preparations were not necessary except for the TEM measurements. TEM samples were prepared first by carbon coating and then FIB was utilized to prepare thin lamellae (in different directions). Preparation of lamellae involved several steps: first, the region of interest on the spicule was coated with up to $3 \mu \mathrm{m}$ of platinum by the use of both electron-beam deposition and ion-beam deposition within the FIB vacuum chamber. Next, each lamella was further cut and thinned so that TEM specimens could be lifted off the sample holder. During the lifting process each lamella was transferred to a molybdenum TEM grid for further thinning with a gallium ion beam 
In the micron-scale we observed a continuous structural pattern from the macro down to the micro, nano, and atomic scales. We show that the spicules demonstrate a unique architecture composed of micron-sized, hexagonally faceted thorns organized in partial spirals along the cylinder-like polycrystalline body of the spicule, and tilted from it at an angle of about $26^{\circ}$. This morphological orientation coincides with the crystallographic orientation relationship between each thorn and the polycrystals within the spicule. Hence the entire spicule grows along the [011] direction of vaterite while the individual thorns grow along the [001] direction. This, together with the presence of both inter- and intracrystalline organic phases, beautifully displays the organism's ability to achieve perfect control of mineralization biologically while employing an unstable polymorph of vaterite. In the nano and atomic scales we found that these spicules are in fact an organic/inorganic nano-composite. Using state-of-the-art aberration-corrected highresolution transmission electron microscopy, we show here that the reason for the failure to explain all the experimental findings by attributing a single and unique crystallographic structure to vaterite, is that this polymorph is composed not of one but at least two different crystallographic structures, which coexist within a pseudo-single crystal (Fig.1) [2]. The major structure demonstrates a hexagonal symmetry; the minor structure, present as different nano-domains within the major matrix, is as yet unknown[3].

Reference:

[1] J. Aizenberg, A. Tkachenko, S. Weiner, L. Addadi, G. Hendler, Nature 412, (2001), 819.

[2] L. Kabalah-Amitai, B. Mayzel, Y. Kauffmann, A.N. Fitch, L. Bloch , P. Gilbert and B. Pokroy. Science (2013) In Press.

[3] The authors are grateful for the financial support of the United States-Israel Binational Science Foundation (BSF) Grant \# 2010065. High-resolution powder diffraction measurements described in this paper were carried out at the beam line ID 31 of ESRF (Grenoble, France)

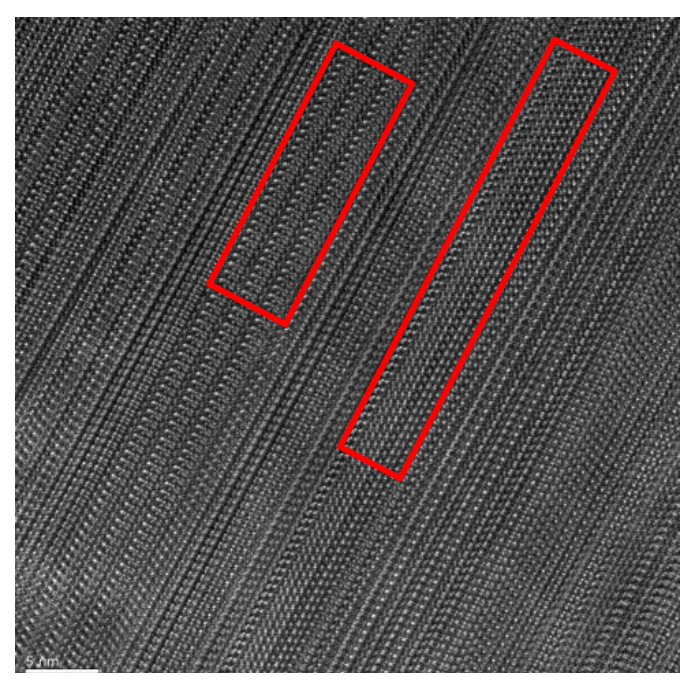

Figure 1. High-resolution transmission electron microscopy image in phase contrast mode. Two distinct atomic orders are marked in red rectangles 